\title{
Clinical Case and Management of a Patient with Gastroenterological Disorders in Type 2 Diabetes Mellitus
}

\section{Yu V Chychula*}

Bogomolets National Medical University, Kyiv, Ukraine

*Corresponding Author: Yu V Chychula, Bogomolets National Medical University, Kyiv, Ukraine.
Received: September 06, 2021

Published: October 25, 2021

(C) All rights are reserved by Yu V Chychula.

\begin{abstract}
Both Diabetes mellitus (DM) and viral hepatitis are relevant when it comes to the discussion of medical and social health problems. Annually worldwide, the number of patients with type 2 diabetes mellitus (T2DM) increases by 7 million people. Hypoacid conditions and achlorhydria are noted in $60 \%$ of patients with chronic atrophic gastritis. Therefore, in addition to disorders of motor function of the stomach, patients with T2DM develop significant metabolic changes in the mucous membrane of the gastroduodenal zone. Some antioxidants are synthesized and activated by the liver, in particular transferrin, ceruloplasmin. T2DM is a manifestation of the metabolic syndrome associated with insulin resistance and is a generally accepted risk factor for non-alcoholic fatty liver disease. Increased activity of liver enzymes in the blood serum may indicate T2DM acts as extrahepatic manifestations of chronic viral hepatitis C.
\end{abstract}

Hepatitis $\mathrm{C}$ is one of the most dangerous liver diseases of viral etiology, the prevalence of which is growing every year. The peculiarity of chronic viral hepatitis $\mathrm{C}$ the presence extrahepatic manifestations. The frequency of extrahepatic manifestations in chronic hepatitis C varies from $38 \%$ to $74 \%$.

According to modern ideas of pathogenesis, type 2 diabetes mellitus (T2DM) is considered as an extrahepatic manifestation of chronic viral hepatitis $\mathrm{C}$, and chronic viral hepatitis $\mathrm{C}$ is considered as an etiological factor in the development of T2DM.

The prevalence of T2DM in patients with viral liver disease is 6-10 times higher than in the general population.

Many patients with extrahepatic manifestations, which are predominant in the clinical picture, may not suspect that they are carriers of viral infection HCV.

The prevalence of Iron deficiency anemia among the adult population is $2 \%$ - $6 \%$. Recently, the relationship between iron deficiency and metabolic syndrome, prediabetes, and T2DM has been studied. Iron deficiency anemia is much more common in patients with type 2 diabetes mellitus. Iron deficiency can disrupt glucose homeostasis and therefore have a negative effect on glycemic control.

The clinical case presented in the article demonstrates the comorbidity of the lesion of the gastrointestinal tract in a patient with T2DM and is of interest to medical doctors.

Keywords: Type 2 Diabetes; Chronic Atrophic Gastritis; H. pylori; Chronic Viral Hepatitis C; Fecal Calprotectin, Fecal Elastase 1; Iron Deficiency Anemia; Sideropenic Syndrome

\section{Introduction}

Both Diabetes mellitus (DM) and viral hepatitis are relevant when it comes to the discussion of medical and social health prob- lems. Annually worldwide, the number of patients with type 2 diabetes mellitus (T2DM) increases by 7 million people. In addition, there are about 3 million deaths from T2DM complications every 
year [1]. Patients with T2DM often develop diabetic autonomic neuropathy. There are many theories of the development of diabetic polyneuropathy and currently, hyperglycemia is known to be the true pathogenetic factor [2-4]. Studies of the effects of the vagus nerve in patients with T2DM indicate the suppression of both pancreatic and gastric secretion. In patients with long-term decompensation of carbohydrate metabolism, the onset of T2DM is followed by hypersecretion of hydrochloric acid. As the disease progresses, there is a decrease in acid-forming and enzymatic functions of the stomach. Hypoacid conditions and achlorhydria are noted in $60 \%$ of patients with chronic atrophic gastritis. Hyperglycemia is known to inhibit gastric secretion. Therefore, in addition to disorders of motor function of the stomach, patients with T2DM develop significant metabolic changes in the mucous membrane of the gastroduodenal zone such as diabetic gastropathy and chronic atrophic gastritis, which are accompanied by hypoxia, impaired acid production, and secretory functions of the stomach [5].

Oxidative stress is considered to be the fundamental mechanism in the pathogenesis of all complications of T2DM. Because some antioxidants are synthesized and activated by the liver, in particular transferrin, ceruloplasmin, their activity depends to some extent on the functional state of hepatocytes. Ceruloplasmin is required for the transport of iron from tissues and its incorporation into transferrin [5].

It should also be noted that $\mathrm{T} 2 \mathrm{DM}$ is a manifestation of the metabolic syndrome associated with insulin resistance and is a generally accepted risk factor for non-alcoholic fatty liver disease. Increased activity of liver enzymes in the blood serum in T2DM patients may indicate not only steatohepatitis but often T2DM acts as extrahepatic manifestations of chronic viral hepatitis C [6].

Hepatitis $\mathrm{C}$ is one of the most dangerous liver diseases of viral etiology, the prevalence of which is growing every year. In addition, hepatitis $\mathrm{C}$ is characterized by a high potential to turn into a chronic stage [4]. The World Health Organization estimates that about 1.75 million people contracted the hepatitis C virus (HCV) in 2016. The main clinical form of HCV infection is chronic viral hepatitis C, which develops in 60 - 80\% of HCV-infected individuals. In such patients, the risk of developing cirrhosis of the liver is 15 - 30\%, of which 4\% may develop hepatocellular carcinoma (HCC) [5-9]. The peculiarity of chronic viral hepatitis $\mathrm{C}$ lies not just in liver impairment alone, but it also affects other internal organs with the predominance of their extrahepatic manifestations in the clinical picture. The frequency of extrahepatic manifestations in chronic hepatitis $\mathrm{C}$ in different countries varies from $38 \%$ to $74 \%$.
According to modern ideas of pathogenesis, type 2 diabetes mellitus (T2DM) is considered as an extrahepatic manifestation of chronic viral hepatitis $\mathrm{C}$, and chronic viral hepatitis $\mathrm{C}$ is considered as an etiological factor in the development of T2DM. A possible confirmation of this is the fact that the nuclear protein of the hepatitis $C$ virus disrupts the insulin reactions cascade [10-12].

The prevalence of T2DM in patients with viral liver disease (chronic hepatitis and cirrhosis) is 6-10 times higher than in the general population $[6,14]$.

These facts suggest that many patients with extrahepatic manifestations, which are predominant in the clinical picture, may not suspect that they are carriers of viral infection $[7,12]$.

Iron deficiency anemia is a main cause of hypoxia as well as an independent risk factor that can lead to death in patients with T2DM which is associated with an increased risk of diabetic complications $[5,13]$.

According to various authors, the prevalence of Iron deficiency anemia among the adult population is $2 \%-6 \%$ and depends on gender, age, race, as well as the presence of occupational hazards and chronic diseases [13]. Recently, the relationship between iron deficiency and metabolic syndrome, prediabetes, and T2DM has been studied. It was found that iron deficiency anemia and manifestations of sideropenic syndrome are much more common in patients with type 2 diabetes mellitus in contrast to the non-diabetic population and averages at 13\% [5]. Iron deficiency can disrupt glucose homeostasis and therefore have a negative effect on glycemic control.

The following clinical case reflects the comorbidity of the lesion of the gastrointestinal tract in a patient with T2DM and is of interest to medical doctors.

\section{Case Report}

Patient M, 44 years old, was hospitalized in the rehabilitation unit for patients with cardiovascular pathology in the Kyiv Clinical Endocrinology Center to correct the hypoglycemic therapy of T2DM. The patient complained of hyperglycemia (fasting glycemia 18 - $22 \mathrm{mmol} / \mathrm{l}$ ), difficulty in selecting adequate hypoglycemic therapy with and without the participation of a family doctor in the prehospital stage. The other symptoms were polyuria, severe weakness, drowsiness, dizziness, and discomfort in the epigastrium and sulfur burps. Periodically, after not following the diet, the patient had pasty stools without pathological impurities up to twice a day; denies weight loss. 
According to the anamnesis, the patient has been suffering from chronic HCV (chronic hepatitis C) since 2005 and thinks that the viral infection is associated with dental treatment. He denies other possible ways of contracting infection such as drug abuse, surgery, getting a tattoo, or blood transfusion. He has been suffering from diabetes since 2006 and was initially receiving insulin therapy up to $250 \mathrm{IU} /$ day. For the last 6 months, he has been received metformin $1000 \mathrm{mg}$ twice a day, but it did not produce effective glycemic control.

During the physical examination, pallor integuments and ictericity of the sclera were noticed. Skin turgor is normal. Peripheral lymph nodes are not enlarged. Body mass index $-23.5 \mathrm{~kg} / \mathrm{m}^{2}$.

Laboratory results confirm hyperglycemia of 16.0-13.5-11.3 mmol/l; glycated hemoglobin 9.2\% (normal 4.5 - 6.5\%); C-peptide 2.85 (normal $1.1-4.4 \mathrm{ng} / \mathrm{ml}$ ).

Blood work results showed decreased hemoglobin level (hemoglobin $111 \mathrm{~g} / \mathrm{l}$ ) and color index (0.71) with a normal number of erythrocytes and other indicators. No clinical picture or history of blood loss episodes.

According to table 1, laboratory test results showed increased activity of some of the liver enzymes.

\begin{tabular}{|l|c|c|c|}
\hline Test Name & Results & Units & $\begin{array}{c}\text { Reference } \\
\text { Range }\end{array}$ \\
\hline $\begin{array}{l}\text { Alanine aminotransfer- } \\
\text { ase (ALT) }\end{array}$ & 66 & $\mathrm{U} / \mathrm{L}$ & up to 45 \\
\hline $\begin{array}{l}\text { Aspartate aminotrans- } \\
\text { ferase (AST) }\end{array}$ & 37 & $\mathrm{U} / \mathrm{L}$ & up to 35 \\
\hline Urea & 5.1 & $\mathrm{mmol} / \mathrm{L}$ & $2.8-7.2$ \\
\hline Creatinine & 80 & $\mathrm{mmol} / \mathrm{L}$ & $71-115$ \\
\hline Bilirubin & 7.54 & $\mu \mathrm{mol} / \mathrm{L}$ & $2.0-21.0$ \\
\hline$\gamma$-glutamate transferase & 23 & $\mathrm{U} / \mathrm{L}$ & $8-61$ \\
\hline alkaline phosphatase & 60 & $\mathrm{U} / \mathrm{L}$ & $40-129$ \\
\hline total protein & 78.3 & $\mathrm{~g} / \mathrm{L}$ & $66-87$ \\
\hline albumin & 49.3 & $\mathrm{~g} / \mathrm{L}$ & $35-52$ \\
\hline albumin & 62.96 & $\%$ & $53-63$ \\
\hline globulins & 37.04 & $\%$ & $37-47$ \\
\hline albumin-globulin ratio & 1.7 & - & $1.0-2.0$ \\
\hline lipid profile & Normal & - & - \\
\hline
\end{tabular}

Table 1: Clinical laboratory report.

Based on laboratory results, additional laboratory and instrumental examinations were conducted.
To identify the cause of iron deficiency anemia, the patient was examined for celiac disease by determining antibodies to deaminated peptides of gliadin, tissue transglutaminase, and exocrine insufficiency of the pancreas. The exocrine insufficiency was assessed based on the level of fecal elastase 1 . In addition, stool examination and blood work were conducted to screen for possible intestinal parasites and the presence of antibodies to amoebae, helminths, and Yersinia, respectively.

The level of fecal calprotectin was determined to exclude inflammatory bowel disease as one of the causes of anemia:

- $\quad$ Pancreatic fecal elastase 1 - $124.34 \mu \mathrm{g} / \mathrm{g}$ in feces (normal > $200 \mu \mathrm{g} / \mathrm{g})$.

- Fecal calprotectin is $7.96 \mu \mathrm{g} / \mathrm{g}$ (normal $<50 \mu \mathrm{g} / \mathrm{g}$ ).

According to the stool examination report, vegetative forms and cysts of protozoa are not detected.

Antibodies to Yersinia, helminths, and deaminated peptides gliadin, tissue transglutaminase are negative.

Esophagogastroduodenoscopy report revealed Erythematous gastroduodenopathy. Biopsy samples were obtained.

Histopathological examination of biopsies showed 2 fragments of the mucous membrane of the antrum of the stomach with signs of chronic active atrophic gastritis (degree of atrophy of $60 \%$ and $70 \%$ of mucosecretory glands, focal intestinal epithelial metaplasia, and reactive foveolar hyperplasia, focal dysplasia/intraepithelial epithelial neoplasia, reduction of mucus secretion, $H$. pylori $(1+)$; some fragments of the mucous membrane of the corner of the stomach show signs of chronic active atrophic gastritis (degree of atrophy - $60 \%$ of mucosecretory glands), reactive foveolar hyperplasia of the mucous membrane; fragments of the gastric mucosa located in the body of the stomach show signs of chronic active atrophic gastritis (degree of atrophy of $40 \%$ and $60 \%$ of oxyntic glands), hyperplasia of the lymphoid follicle. Aas 3, Cas 2, OLGA stage IV. Helicobacter pylori (1+).

Histopathological examination showed chronic active metaplastic atrophic gastritis B-type associated with H.pylori. Focal dysplasia/intraepithelial neoplasia of epithelium of the antrum of the stomach, mild level. OLGA stage IV atrophy, stage II inflammatory activity (according to Aruin) (Photo 1).

Ultrasound examination of the abdominal cavity indicated the presence of gallbladder polyps, chronic acalculous cholecystitis; diffuse changes in the liver. 


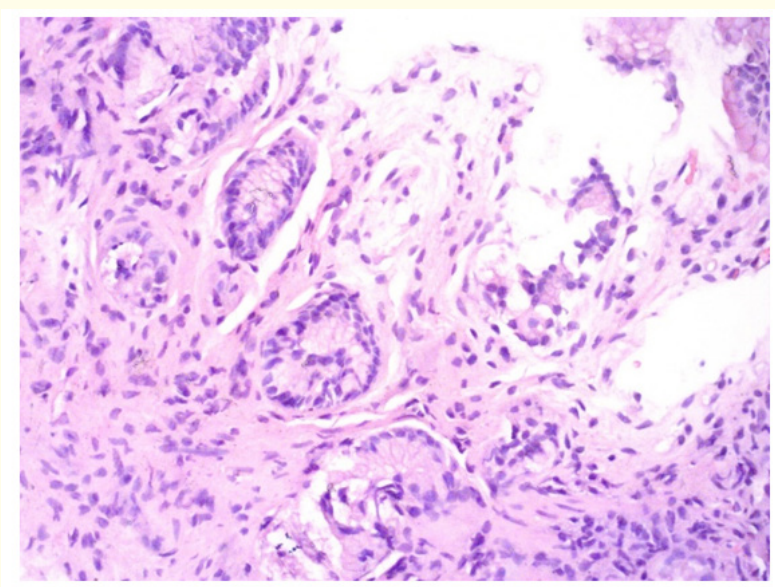

Photo 1: The mucosa of the antrum of the stomach with atrophy of the glands, focal lympho-plasmacytic cell infiltration with impurities of polymorphonuclear leukocytes. Hematoxylin and eosin staining. Magnification x100.

Videocolonoscopy was performed to further differential diagnostics of Sideropenic syndrome under intravenous anesthesia. The device was inserted into the dome of the cecum. The lumen, haustration, color, and vascular pattern of the mucosa are normal, correspond to the departments. There are single flat polypoid formations up to $0.5 \mathrm{~cm}$ with unchanged mucosa in the descending colon. Biopsy samples were obtained. No other changes in the intestinal mucosa were detected. Conclusion: Focal hypertrophy of the sigmoid mucosa of the colon.

Based on histopathological examination of a biopsy of the colon: areas of mucosa with hyperplasia of the glands such as acinar adenoma; submucosal layer is absent.

Detailed diagnosis of Sideropenic syndrome revealed a decrease in serum iron to $3.1 \mu \mathrm{mol} / \mathrm{l}$ (norm 11.6 - $31.3 \mu \mathrm{mol} / \mathrm{l}$ ), ferritin to $8.40 \mathrm{ng} / \mathrm{ml}$ (norm $22-322 \mathrm{ng} / \mathrm{ml}$ ), and a decreased saturation of transferrin with iron down to $8.9 \%$ at a rate of $20-55 \%$. Levels of folic acid, cyanocobalamin were detected within normal limits. Transferrin values are $3.6 \mathrm{~g} / \mathrm{l}$ within normal limits (2.15 - $3.6 \mathrm{~g} / \mathrm{l})$.

Therefore, given the absence of a visible source of blood loss, absorption disorders associated with the presence of chronic atrophic gastritis in patients with chronic viral hepatitis C and T2DM are suspected to be a possible cause of chronic iron deficiency anemia.

Polymerase chain reaction (PCR) (qualitative and quantitative) was conducted to confirm the viral etiology of the pathology of the liver and for genotyping. Qualitative PCR showed the presence of hepatitis C RNA. The 1 b genotype of HCV was also identified. Quantitative PCR of hepatitis $C$ virus exceeded the analytical sensitivity of the test system (linear range 12-1.0 $108 \mathrm{IU} / \mathrm{ml}$ ) and was 6.26 $\cdot 106 \mathrm{IU} / \mathrm{ml}$.

As noted, the natural course of chronic HCV invariably leads to fibrosis liver cirrhosis and hepatocellular carcinoma. Therefore, to study the stage of fibrosis the patient underwent Transient elastography and liver steatometry.

Elastography and steatometry revealed: Chronic hepatitis, moderate. F3 by elastography (METAVIR), steatometry S.1. Chronic cholangitis of the intrahepatic bile ducts. Chronic acalculous cholecystitis without exacerbation. Cholesterolisis of the gallbladder, polyposis-reticular form, pronounced. Hypotension of the gallbladder, moderate severity. Chronic pancreatitis, moderate severity. Splenomegaly is insignificant.

Therefore, after taking into account the complaints, history, objective examination, and the results of laboratory and instrumental methods, the final clinical diagnosis was established: Type 2 diabetes mellitus (T2DM). Chronic viral hepatitis C, fibrosis stage F3 (according to METAVIR), steatometry S.1. Chronic acalculous cholecystitis, cholesterolisis of the gallbladder. Chronic pancreatitis with exo- and endocrine insufficiency. Chronic metaplastic atrophic gastritis B associated with H. pylori. Diabetic gastroparesis. Mild iron deficiency anemia, Sideropenic syndrome. Hypertrophic polyps of the sigmoid colon.

The patient underwent a course of antihelicobacter triple therapy pantoprazole $40 \mathrm{mg}+$ amoxicillin $1000 \mathrm{mg}+$ clarithromycin $500 \mathrm{mg}$ (twice daily) for 10 days with eradication control 1 month after the end of treatment, which was achieved successfully. Correction of dyspeptic symptoms and gastroparesis was performed by prescribing itopride hydrochloride $50 \mathrm{mg}$ before meals for 20 days.

To correct iron deficiency, the patient was prescribed iron sulfate in combination with ascorbic acid for 3 months. After treatment with iron supplements, positive clinical and laboratory dynamics were obtained:

- $\quad$ Iron $27.8 \mu \mathrm{mol} / \mathrm{l}$ (norm 11.6 - $31.3 \mu \mathrm{mol} / \mathrm{l}$ ).

- Ferritin $28.5 \mathrm{ng} / \mathrm{ml}$ (norm 22 - $322 \mathrm{ng} / \mathrm{ml}$ ).

The patient was also seen by an infectious disease specialist and it was recommended to prescribe a pan-genotypic drug with direct antiviral action (combined drug sofosbuvir $400 \mathrm{mg}+$ velpatasvir 
$100 \mathrm{mg}$ per day) for 3 months. After 1 month from the start of treatment, a decrease in viral load was observed. After 3 and 6 months, the RNA of the hepatitis C virus was not detected.

Hypoglycaemic therapy was adjusted with the combination of empagliflozin $12.5 \mathrm{mg}$, metformin $1000 \mathrm{mg}$, and glimepiride $2 \mathrm{mg}$ in the morning and metformin $1000 \mathrm{mg}$ in the evening, which significantly improved fasting glucose and glycated hemoglobin.

Correction of external secretory insufficiency of the pancreas was performed in the form of enzyme replacement therapy with 25,000 pancreatin minimicrospheres 25,000 during each meal for a long period.

The course of diseases of the gastrointestinal tract in patients with T2DM has some specific features.

In patients with type 2 diabetes, HCV may increase insulin resistance, which complicates the process of carbohydrate metabolism compensation and requires correction. HCV has many extrahepatic manifestations, which usually do not affect the progression of the process in the liver, but affect the quality of life of patients and are predictors of adverse responses to antiviral therapy.

Patients with chronic atrophic gastritis, T2DM, and other comorbid pathologies can quickly develop iron deficiency anemia with clinical and laboratory manifestations of the sideropenic syndrome.

\section{Conclusion}

This presented clinical case demonstrate the importance of intime diagnostics of comorbid pathology among patient with type 2 diabetes mellitus and requires careful differential diagnosis and the development of individual schemes of personalized treatment.

\section{Bibliography}

1. WHO. Hepatitis C. Key facts.

2. WHO. GLOBAL HEALTH SECTOR STRATEGY ON VIRAL HEPATITIS 2016-2021, Geneva.

3. Golubovskaya OA., et al. "Correction of polyneuropathy in type II diabetes mellitus in patients with chronic hepatitis C". Clinical Infectiology and Parasitology 1.04 (2013): 141-150.

4. Golubovska O. "Epidemiology and a natural curse of a virus $\mathrm{C}$ hepatitis". Clinical Endocrinology and Endocrine Surgery 4.25 (2008): 48-51.
5. Broide E., et al. "Expression of Duodenal Iron Transporter Proteins in Diabetic Patients with and without Iron Deficiency Anemia". Journal of Diabetes Research 6 (2018): 7494821.

6. Younossi Z., et al. "Extrahepatic Manifestations of Hepatitis C: A Meta-analysis of Prevalence, Quality of Life, and Economic Burden". Gastroenterology 150.7 (2016): 1599-1608.

7. Cacoub P., et al. "Extrahepatic manifestations of chronic hepatitis C virus infection". Therapeutic Advances in Infectious Disease 3.1 (2016): 3-14.

8. Durand F and Valla D. "Assessment of prognosis of cirrhosis". Seminars in Liver Disease 28.1 (2008): 110-122.

9. El-Serag HB. "Epidemiology of viral hepatitis and hepatocellular carcinoma”. Gastroenterology 142.6 (2012): 1264-1273. e1.

10. Global hepatitis report. Geneva: World Health Organization (2017).

11. Hui JM., et al. "Insulin resistance is associated with chronic hepatitis C and fibrosis progression". Gastroenterology 125 (2003): 1695-1704.

12. Mason AL., et al. "Association of diabetes mellitus and chronic hepatitis C virus infection". Hepatology 29 (1999): 328-333.

13. Merlo ChM and Wuillemin WA. "Prevalence and causes of anemia in a city general practice". Praxi 97.13 (2008): 713-718.

14. Younossi ZM., et al. "Hepatitis C infection: a multifaceted systemic disease with clinical, patient reported and economic consequences". The Journal of Hepatology 65.1 (2016): S109S19.

\section{Volume 4 Issue 11 November 2021 (C) All rights are reserved by Yu V Chychula.}

